p. 396-397 ; L, p. 490-494; LI, p. 123-125, p. 380-381 ; LII, p. 221-222, p. 474475 ; LIII, p. 166-172, p. 287; LIV, p. 567-575; LV, p. 72-75, p. 228-232, p. 494-498).

M. Lutz donne lecture de la communication suivante :

\title{
Sur les Pédiculaires de la Savoie;
}

\author{
PAR M. ALFRED CHABERT.
}

L'extrème désinvolture avec laquelle Huguenis préparait ses centuries et ses envois de plantes, a causé et cause encore de nombreuses erreurs relatives à la flore de la Savoie. Les botanistes qui n'en sont pas avertis le citent et parfois même ceux qui le sont l'oublient et, trompés par des échantillons faussement indiqués comme recueillis dans une localité, se laissent aller à écrire, au sujet d'une détermination exacte d'un des plus savants botanistes du siècle dernier, Reichenbach : « err. infaustum nomen ".

Les erreurs que je crois devoir relever aujourd'hui, pour épargner des déceptions aux confrères chaque année plus nombreux qui viennent herboriser dans nos riches montagnes, concernent les six Pédiculaires suivantes :

$\times P$. Huguenini Rchb. f., indiqué au mont Margériaz.

$\times P$. Penzigii Steing, indiqué au mont Granier.

$\times P$. Rouyana F. O. Wolf, idem.

$P$. cenisia Gaud., idem.

P. tuberosa L., idem.

$P$. incarnata Jacq., idem.

Le mont Margériaz et le mont Granier ou Grenier sont deux montagnes calcaires, hautes la première de 1841 mètres, la

1. Faisant un commerce considérable de plantes d'herbier, Huguenis en tenait un grand magasin sans cesse renouvelé, où il intercalait pêlemêle dans les mêmes feuilles les plantes qu'il récoltait et celles qu'il recevait, pourvu qu'elles eussent entre elles une certaine ressemblance ou que leurs étiquettes portassent le même nom. Ensuite, pour ses envois, il puisait au hasard dans la feuille un ou plusieurs échantillons et, sans se préoccuper de leur origine réelle, il y joignait une de ses étiquettes imprimées ou manuscrites préparées à l'avance. C'est ainsi que j'ai vu dans plusieurs herbiers le Pedicularis cenisia Gaud. indiqué sur les montagnes des environs de Chambéry sous le nom de gyroflexa, etc. 
seconde de 1940, situées l'une à l'est, l'autre au sud de Chambéry ${ }^{1}$ et appartenant la première au massif des Bauges, la seconde à celui de la Chartreuse. Ainsi que Songeos et moi l'avons indiqué en 1896, dans nos Herborisations aux environs de Chambéry, p. 29 et 37, le Pedicularis gyroflexa Vill. est commun sur le Margériaz; le Grenier nourrit en abondance les $P$. gyroflexa et Barrelieri Rchb. aussi bien sur les pentes au-dessus d'Entremont qui appartiennent à la Savoie que sur le plateau qui fait partie du département de l'Isère. La présence du Barrelieri sur ce plateau et sur les montagnes de l'Arpetta et de l'Aut du Chu ${ }^{2}$ qui lui font suite et appartiennent au même département, avait déjà été signalée par moi le 11 mars 1859 à la Société botanique de France et publiée dans son Bulletin, confirmant ainsi l'adjonction douteuse jusqu'alors de cette plante à la flore de France.

Mais ni les $P$. cenisia, tuberosa, incarnata ni aucun autre ne se trouvent sur le Margériaz et le Grenier; ils n’y ont jamais été signalés qu'à la suite des incorrections d'Huguesin ou des déterminations inexactes de jeurres botanistes. La station du tuberosa la plus rapprochée de Chambéry est dans les Alpes de Saint-Hugon au-dessus de La Rochette, de l'autre côté de la vallée de l'Isère; celles des cenisia et incarnata sont à plus grande distance, dans nos hautes Alpes de Maurienne et de Tarantaise.

Pedicularis Huguenini Rchb. fil. Icon. fl.germ. et helv. 20, p. 73, n. 10, t. 1777, Mont Margériaz (Huguenin, sec. Rchb.), n'existe pas plus sur cette montagne que les cenisia et incarnata, dont il serait un hybride d'après M. Rour Fl. Fr., XI, p. 113. C'est une plante fort douteuse qui a été rapportée au gyroflexa par Steninger, Beschr. der Europ. Arten des Genus Pedicularis (Bot. Centralblatt, 1887, p. 8) et qui n’a pas été retrouvée. Rien de ce que j'ai vu sous le nom d'Huguenini dans l'herbier Borssier et dans d'autres collections n'est conforme à la diagnose ni à la table de Reichesbach. Il manque à l'herbier d'Huguenis. Si sa genèse par cenisia $\Varangle$ incarnata est réelle, il n'est pas impossible

1. L'exploration de chacune d'elles est assez pénible, mais peut se faire dans la journée depuis Chambéry.

2. L'Aut du Chu est indiqué dans les cartes actuelles sous le nom de "Haut du Seuil "; c'est une traduction erronée du patois local. Aut du Clın ou Au dechu signifie : au-dessus. 
qu'il se rencontre ailleurs en Savoie; car je me souviens qu'herborisant en aoùt 1876 avec Soxgeox dans les Alpes de Velmeinier, il recueillit, non loin de Notre-Dame-des-Neiges, un individu qui nous a paru un hybride des deux espèces susdites. Malheureusement il ne l'a pas conservé, et il n'est pas dans son herbier. La plante décrite par Reichenвach fils peut donc bien avoir été récoltée quelque part dans nos Alpes; mais pour qui a vu Huglens préparer ses envois, il est tout aussi admissible qu'elle l'ait été dans celles de Suisse, de Piémont ou de Ligurie. Du reste Huguesix fut bien puni par où il avait péché! Jamais il ne put retrouver la Pédiculaire portant son nom, malgré l'argent qu'il dépensait chaque été pour faire recueillir par le Grand Pendiculaire ${ }^{1}$ sur Margériaz et Grenier des masses de ces plantes au risque de les détruire. Jamais il n'en parla à Soxgeox ni à moi, qui n'en ai connu l'existence que par l'ouvrage de Steisivger en 1887 ; mais il nous parlait beaucoup d'une autre plante trouvée une seule fois par lui sur le Granier, qu'il avait baptisée : $P$. gyroflexa var. grossa, et qui échappa à toutes les recherches ultérieures.

P. Penzigii Steingr, l. c. p. 186.

P. tuberosa $\times$ fasciculata Muret, Mont Granier (Huguenin sec. Steininger). N'a jamais pu être recueilli sur cette montagne où manquent les $P$. tuberosa, fasciculata, cenisia et incarnata auxquels Muret, M. Pexzig, Carcel et M. Rouy ont successivement attribué sa genèse adultérine. C'est, comme l'a fort bien dit Muret, un luberosa $\times$ fasciculala". Je l'ai découvert en Savoie,

1. Vieux grenadier de la Garde Impériale, haut de plus de deux mètres, ayant fait toutes les guerres de la Révolution et de l'Empire, couvert de blessures, il avait pris goùt à la botanique chez un médecin allemand qui le soigna après Bautzen d'un coup de lance à la jambe et l'employa durant sa convalescence à changer de papier les plantes de son herbier. Rentré dans la vie civile, il passait la belle saison à récolter des herbes pour les pharmaciens et surtout des plantes pour Bonjean et Huguenis. Bien payé par celui-ci pour les Pédiculaires, il en demandait à tous les paysans des montagnes. De là le surnom de "Grand Pendiculaire" qui lui fut donné par eux, et plus tard celui de "Pendiculaire ", qu'ils attribuèrent long. temps aux botanistes herborisant.

1. Le $P$. fasciculata Bell. confondu par quelques auteurs avec le gyroRexa Vill. en est bien différent. Il n'est pas rare sur le mont Cenis et sur nos Alpes voisines. 
le 9 aoùt 1878, dans les prairies alpines du Col des Encombres à la base du Grand Perron, au milieu de ses parents, et n'ai pu le revoir les années suivantes.

Pedicularis Rouyana F. O. Wolf ap. Rouy Illustr. pl. Eur. var. 17 p. 137, t. 416; P. cenisia $\times$ tuberosa F. O. Wolf., l. c., Mont Granier (Huguenin sec. Rouy, Fl. fr., XI, p. 120). Indication inexacte, en raison de l'absence des parents.

P. delphinata Steingr, l. c., p. 37; P. gyroflexa $\times$ Barrelieri Rchb. (err. infaustum nomen Rouy, l. c.). P. cenisia! $\times$ Barrelieri Rouy, l. $c$. Mont Granier, près de la montée de l'Arpetta (Herb. J. Gay, sec. Rocy l. c.) L'indication est exacte. La plante a été trouvée vers 1834 par Bonjean, qui la distribuait avec des étiquettes imprimées portant le $\mathrm{n}^{\circ} 34$ et les indications suivantes : P. gyroflexa B. leucantha. Pédiculaire à fleurs blanches, vivace. Montagne de Grenier, près la montée de l'Arpetta. Cette variété a été observée pendant cinq années. Fl. juillet. »J'en ai vu et recueilli un seul individu, en aoùt 1852, non loin de la Caverne du Granier, localité voisine de celle de Bonjean, au milieu des gyroflexa et Barrelieri, les seules Pédiculaires, comme je l'ai dit, qui croissent sur cette montagne. C'est donc bien un gyroflexa $\times$ Barrelieri, comme l'a dit Reichenвach, et non un cenisia $\times$ Barrelieri, comme le veut $M$. Rouy, et si l'épithète " err. infaustum nomen » devait être conservée, ce ne serait certainement pas pour le binome du célèbre botaniste allemand.

P. Vulpii Solms-Laub. in Oest. bot. Zeit., 15, p. 174; P.incarnata $\times$ tuberosa Vulp. in Flora, 1854, p. 97. J'en ai revu, le 14 août dernier, une dizaine d'individus dans les prairies alpines du Galibier, au-dessus des Granges.

A propos de cette communication, M. Rouy fait quelques observations qu'il se propose de développer brièvement à la première séance de novembre.

Il est donné connaissance d'une Étude de M. Dubard sur le genre Palaquium. Ce travail sera l'objet du Mémoire $\mathrm{n}^{\circ} 16$. 


\section{$2 \mathrm{BHL}$ Biodiversity Heritage Library}

Chabert, Alfred. 1909. "Sur les Pédiculaires de la Savoie." Bulletin de la Société botanique de France 56, 499-502.

https://doi.org/10.1080/00378941.1909.10832088.

View This Item Online: https://www.biodiversitylibrary.org/item/8678

DOI: https://doi.org/10.1080/00378941.1909.10832088

Permalink: https://www.biodiversitylibrary.org/partpdf/160822

\section{Holding Institution}

Missouri Botanical Garden, Peter H. Raven Library

\section{Sponsored by}

Missouri Botanical Garden

\section{Copyright \& Reuse}

Copyright Status: Public domain. The BHL considers that this work is no longer under copyright protection.

This document was created from content at the Biodiversity Heritage Library, the world's largest open access digital library for biodiversity literature and archives. Visit BHL at https://www.biodiversitylibrary.org. 